\title{
Enhancing test precision for local Lorentz symmetry violation with entanglement
}

\author{
Lei Li, ${ }^{1}$ Xinwei Li, ${ }^{2}$ Baocheng Zhang,,$*$ and $\mathrm{Li} \mathrm{You}^{2}$ \\ ${ }^{1}$ School of Mathematics and Physics, China University of Geosciences, Wuhan 430074, China \\ ${ }^{2}$ State Key Laboratory of Low Dimensional Quantum Physics, \\ Department of Physics, Tsinghua University, Beijing 100084, China
}

\begin{abstract}
A recent proposal for testing Lorentz symmetry violation (LSV) presents a formulation where the effect of violation is described as a local interaction [R. Shaniv, et al, Phys. Rev. Lett. 120, 103202 (2018)]. An entangled ion pair in a decoherence free subspace (DFS) is shown to double the signal to noise ratio (SNR) of one ion, while (even)-N/2 such DFS pairs in a collective entangled state improve SNR by $\mathrm{N}$ times, provided the state parity or the even/odd numbers of ions can be measured. It remains to find out, however, how such fiducial entangled states can be prepared at nonexponentially small success rates. This work suggests two types of many particle entangled states for testing LSV: the maximally entangled NOON state, which can achieve Heisenberg limited precision; and the balanced spin-1 Dicke state, which is readily available in deterministic fashion. We show that the latter also lives in a DFS and is immune to stray magnetic fields. It can achieve classical precision limit or the standard quantum limit (SQL) based on collective population measurement without individual atom resolution. Given the high interests in LSV and in entanglement assisted quantum metrology, our observation offers additional incentives for pursuing practical applications of many atom entangled states.
\end{abstract}

\section{INTRODUCTION}

Invariance under Lorentz transformation constitutes one of the most fundamental principles of modern physics. A more complete theory including quantum gravity, however, implies the possibility or even necessity for Lorentz symmetry violation (LSV) [1-3]. Hence, the study of this violation has attracted wide attention. Over the past twenty years a theoretical framework called the Standard-Model Extension (SME) [4 $[6]$ is developed, which formally contains all possible symmetry breaking terms generated by couplings between the standard fields and the vacuum expectations of the tensor fields parameterizing symmetry violations. Within the SME, the symmetry breaking terms collectively do not cause LSV in the Planck scale. But in low energy scale (due to spontaneously symmetry breaking) every term causes violation in the presumed absence of the others [5], and can be studied experimentally.

Of direct relevance to this study concerns several recent efforts using atoms or ions to test LSV through precision measurements. The specific LSV term usually concerns the isotropy of the speed of light (for recent results, please refer to Ref. (7]), with the most sensitive tests to date using neutral Dy atoms [8], $\mathrm{Ca}^{+}$ions [9], and $\mathrm{Yb}^{+}$ions [10]. An alternative proposal recently suggests testing atomic level spacing with atomic clock 11] (augmented by dynamic decoupling), predicting an extremely high sensitivity to LSV. Yet another term in the SME concerns the dependence on the direction of motion and the momentum of a particle, which also violates local Lorentz invariance (LLI) [12]. If the total angular momentum of a physical system is fixed, this latter term becomes propor-

*Electronic address: zhangbc.zhang@yahoo.com tional to the square of the $z$-component of the total angular momentum operator, as in quadratic Zeeman shift for an atomic hyperfine spin, which constitutes a typical local interaction [11].

The first two types of tests mentioned above all reduce to atomic clock measurements. Their precisions can reach beyond the classical precision limit, or the standard quantum limit $(\mathrm{SQL})(\propto 1 / \sqrt{N})$, when ensembles of entangled particles are employed [13]. Our studies indicate that the third type of term, an equivalent quadratic Zeeman shift or a local interaction form, can also be tested beyond the SQL with entangled particles.

This paper is organized as follows. First we introduce the relevant background as well as the formulation for the specific LSV term (from the SME) we study. This is followed by a discussion of the recent experimental proposal for estimating its strength with the help of a DFS. Our main contribution is then presented by suggesting two types of many atom entangled states instead. First, the maximally entangled NOON state, with which Heisenberg limited (HL) precision $\propto 1 / N$ becomes possible; Second, the balanced spin-1 Dicke state [14], which lives in its own DFS and is thus also immune to stray magnetic fields, can be employed to reach the SQL based on collective population measurement without requiring individual atom resolution.

\section{LORENTZ SYMMETRY VIOLATION}

Based on the modern description of nature at the most fundamental level, Lorentz symmetry might be violated at experimentally accessible energy scales due to spontaneous symmetry breaking, hence the development of its experimental test [7-11, 15-18]. In relativistic physics, Lorentz symmetry implies an equivalence of observation or observational symmetry according to special relativity, 
which is formally equivalent to stating that the laws of physics stay the same for all observers moving at constant velocities with respect to each other in inertial frames. It is also described as the independence of experimental results on orientation or boost velocity of the apparatus setup through space [12]. When LSV is considered for the electron sector within the framework of SME, the QED Lagrangian (for electron) becomes [4, 5],

$$
L=\frac{1}{2} i \bar{\psi}\left(\gamma_{\nu}+c_{\mu \nu} \gamma^{\mu}\right) \overleftrightarrow{D}^{\nu} \psi-\bar{\psi} m_{e} \psi
$$

where $m_{e}$ denotes the electron mass, $\psi$ is a Dirac spinor, $\gamma^{\mu}$ are the usual Dirac matrices and $\bar{\psi} \overleftrightarrow{D^{\nu}} \psi \equiv \bar{\psi} D^{\nu} \psi-$ $\psi D^{v} \bar{\psi}$ with $D^{v}$ the shorthand for the covariant derivative. The $c_{\mu \nu}$ tensor in the above Eq. (11) quantifies the strength of LSV for the electron sector by the frame dependent interaction term, which gives an energy level shift [18 20]: $\delta H_{\mathrm{LSV}}=-\left[C_{0}^{(0)}-2 U c_{00} /\left(3 c^{2}\right)\right] \mathbf{p}^{2} / 2-$ $C_{0}^{(2)} T_{0}^{(2)} / 6$, responsible for the first two types of LSV tests mentioned in the last section. Here, $U$ is the Newtonian gravitational potential and the more specific parameters $C_{0}^{(0)}$ and $c_{00}$ quantifying the strength of LSV have been discussed and tested before [8], but are not required for the following discussions.

The relativistic form of rank 2 irreducible tensor operator $T_{0}^{(2)}$ is $T_{0}^{(2)}=c \gamma_{0}\left(\gamma \mathbf{p}-3 \gamma_{z} p_{z}\right)$, with $p_{z}$ the momentum component along the quantization axis fixed in the laboratory frame. Its non-relativistic form becomes $T_{0}^{(2)}=\left(\mathbf{p}^{2}-3 p_{z}^{2}\right) / m_{e}$. Thus the second LSV term $\propto C_{0}^{(2)}$ of $\delta H_{\mathrm{LSV}}$ reduces to

$$
\delta H=-C_{0}^{(2)} \frac{\left(\mathbf{p}^{2}-3 p_{z}^{2}\right)}{6 m_{e}},
$$

which is responsible for breaking of the symmetry on independence of orientation or velocity in a bound electron system such as a $\mathrm{Ca}^{+}$ion [9]. Its diagonal matrix element

$$
\left\langle j, m\left|T_{0}^{(2)}\right| j, m\right\rangle=\frac{\left[-j(j+1)+3 m^{2}\right]\left\langle j\left|T^{(2)}\right| j\right\rangle}{\sqrt{(2 j+3)(j+1)(2 j+1) j(2 j-1)}},
$$

is calculated using Wigner-Eckart theorem [9], with $j$ and $m$ the corresponding quantum numbers for the total and z-component electron angular momentum. The latter $(\propto$ $m^{2}$ term) corresponds to raw LSV signal. For a physical system with a fixed $j$, such as a bound electron system first suggested in Ref. [11], the LSV dynamics is thus described by an equivalent Hamiltonian $H_{V}=\kappa j_{z}^{2}$, which is analogous to a quadratic Zeeman shift.

In earlier LSV tests of the local interaction term Eq. (2), eigenstates of distinct absolute angular momentum $j_{z}(=m)$ are chosen in order to extract the relative (time) phase from a coherent superposition, over days or even longer times. In Ref. [11], the selected states are $\left|\frac{7}{2},-\frac{7}{2}\right\rangle$ and $\left|\frac{7}{2},-\frac{1}{2}\right\rangle$, their relative time phase is measured by Ramsey interferometry, sometimes, augmented by dynamical decoupling (DD) [21], as described in the following. In terms of the total angular momentum operator for a particle, the time evolution operator for the Ramsey interferometry becomes [22, 23]

$$
U_{\phi}=e^{-i \pi j_{x} / 2} e^{i \phi j_{z}} e^{i \pi j_{x} / 2}=e^{-i \phi j_{y}},
$$

with the free evolution term $e^{i \phi J_{z}}$ (rotation of an angle $\phi$ around the $z$-axis) sandwiched in between two $\pi / 2$ pulses that effectively serve as 50:50 beam splitters. Thus the relative phase $\phi$ maps onto the familiar differential optical path of the Mach-Zehnder interferometry. With the LSV term included into free evolution, the total time evolution changes into

$$
U_{\phi, \kappa}=e^{-i \pi j_{x} / 2} e^{i \phi j_{z}} e^{-i \kappa t j_{z}^{2}} e^{i \pi j_{x} / 2}=e^{-i \phi j_{y}-i \kappa t j_{y}^{2}},
$$

where the second term in the exponent above $\propto \kappa$ resembles the one-axis spin squeezing [24] or its associated quadratic interaction in nonlinear Ramsey interferometry [25 27]. A careful examination reveals, however, that this is simply not the case as the quadratic spin operator $j_{z}^{2}$ here refers to one particle rather than the collective spin of an ensemble [24].

It is well known according to parameter estimation theory [28, 29] that an ensemble of uncorrelated particles, even in the ideal case, provides the best achievable precision for estimating $\delta \kappa \propto 1 / \sqrt{N}$, consistent with the SQL. The total LSV Hamiltonian for an ensemble of $N$ atoms can be expressed as

$$
H_{V}=\kappa\left(j_{z}^{(1)}\right)^{2}+\kappa\left(j_{z}^{(2)}\right)^{2} \cdots+\kappa\left(j_{z}^{(N)}\right)^{2} \equiv \kappa \mathcal{H}
$$

where $j_{z}^{(i)}$ denotes $j_{z}$ for the i-th atom, and $\mathcal{H}=\sum_{i=1}^{N}\left(j_{z}^{(i)}\right)^{2}$ is the generator for estimating $\kappa$. Evidently, the ensemble evolution operator is

$$
U_{\phi, \kappa}=\prod_{i=1}^{N} e^{-i \phi j_{y}^{(i)}-i \kappa t\left(j_{y}^{(i)}\right)^{2}},
$$

which remains local as $H_{V}$ induces no entanglement if all atoms are initially in product states. Nevertheless, various techniques can be implemented for improved estimation of $\kappa$ based on simple population measurements in spin components in the end [1].

Quantum estimation theory [28, 29] allows for the precision of estimating a local parameter to go beyond the SQL, e.g. with quantum entangled ensembles. For the LSV term [11] we discuss, it was pointed out that an entangled pure state of two $\mathrm{Yb}^{+}$ions,

$$
\frac{1}{\sqrt{2}}\left(\left|\frac{7}{2}, \frac{7}{2}\right\rangle\left|\frac{7}{2},-\frac{7}{2}\right\rangle+\left|\frac{7}{2}, \frac{1}{2}\right\rangle\left|\frac{7}{2},-\frac{1}{2}\right\rangle\right)
$$

gives a factor of $\sqrt{2}$ improvement in the signal to noise ratio (SNR), beating the SQL [9]. In addition, such a two-ion state forms a decoherence free subspace (DFS), 
which significantly suppresses stray magnetic fields. Extrapolating further (in the supplemental materials of Ref. [9]), an analogous paired entangled state

$$
\frac{1}{\sqrt{2}}\left(\left|\frac{7}{2}, \frac{7}{2}\right\rangle^{\otimes \frac{N}{2}}\left|\frac{7}{2},-\frac{7}{2}\right\rangle^{\otimes \frac{N}{2}}+\left|\frac{7}{2}, \frac{1}{2}\right\rangle^{\otimes \frac{N}{2}}\left|\frac{7}{2},-\frac{1}{2}\right\rangle^{\otimes \frac{N}{2}}\right),
$$

is shown to provide $N$-times enhanced precision (for $N$ even). The two states in Eqs. (6) and (7) provide enhanced estimation of $\kappa$, but require parity measurements, i.e., the ability to determine even/odd of the total numbers of ions projected into a specific internal state, although Ramsey interferometries are applied to all ions at once. The required parity measurement, however, represents a serious constrain against the most significant advantages for ensemble based precision measurement: collective manipulations and measurements, with Ramsey pulses applied to all particles at once indiscriminately and total populations in different single particle internal states counted in the end without single atom resolution. In the following, we suggest two alternative multiparticle entangled states for estimating $\kappa$ with enhanced precision.

\section{NOON STATE AND THE BALANCED SPIN-1 DICKE STATE}

The state Eq. (7) is very suggestive, as it represents nothing but an equal superposition of two twinFock states [30]. Each of the twin-Fock modes satisfies $m=-m$, offering the combined advantages of: DFS or immunity (or insensitivity) to stray magnetic fields, and a cumulative relative $m^{2}$-dependent LSV phase. For any meaningfully large $N$, however, it is unclear how such a state can be generated with high yield as the method of Ref. [9 11] is exponentially ineffective.

More generally, estimating $\kappa$ starts with encoding an input state $\rho$ by a unitary transformation $U=e^{-i \kappa t \mathcal{H}}$ and follows with measurements to the output state $\rho_{\kappa}=$ $U \rho U^{\dagger}$. The ultimate precision is given by the quantum Cramér-Rao bound (QCRB) 31, 32]

$$
\delta \kappa \geq \frac{1}{\sqrt{\nu}} \frac{1}{T \sqrt{F_{Q}}}
$$

where $F_{Q}$ denotes quantum Fisher information (QFI) and is determined by the output state $\rho_{\kappa}, T$ is the time duration of an ensemble state (used to probe) under $H_{V}$ and $\nu$ is the number of experimental trials. If the probe state is pure $|\psi\rangle$, QFI simplifies to

$$
F_{Q}=4(\Delta \mathcal{H})^{2}=4\left(\left\langle\mathcal{H}^{2}\right\rangle-\langle\mathcal{H}\rangle^{2}\right),
$$

which gives the QCRB for a pure probe state [33]

$$
\delta \kappa \geq \frac{1}{\sqrt{\nu}} \frac{1}{2 T \Delta \mathcal{H}},
$$

with the variance $(\Delta \mathcal{H})^{2}=\left\langle\mathcal{H}^{2}\right\rangle-\langle\mathcal{H}\rangle^{2}$, bounded by

$$
\Delta \mathcal{H} \leq\left(\Lambda_{\max }-\Lambda_{\min }\right) / 2 .
$$

$\Lambda_{\max }$ and $\Lambda_{\text {min }}$ are the maximum and minimum eigenvalues of $\mathcal{H}$ [31]. This bound can be achieved by using the initial probe state $\left(\left|\Lambda_{\max }\right\rangle+\left|\Lambda_{\min }\right\rangle\right) / \sqrt{2}$, a cat state with $\left|\Lambda_{\max }\right\rangle\left(\left|\Lambda_{\min }\right\rangle\right)$ the eigenstate of corresponding eigenvalue $\Lambda_{\max }\left(\Lambda_{\min }\right)$. The maximum and minimum eigenvalue for $\mathcal{H}$ defined in Eq. (4) is $N \lambda_{\max }$ and $N \lambda_{\min }$ respectively, where $\lambda_{\max }\left(\lambda_{\min }\right)$ denotes the maximum (minimum) eigenvalue of the single particle $\left(j_{z}^{(i)}\right)^{2}$. Thus the QCRB is given by

$$
\delta \kappa \geq \frac{1}{\sqrt{\nu}} \frac{1}{T N\left|\lambda_{\max }-\lambda_{\min }\right|},
$$

which is clearly capable of reaching HL precision $\delta \kappa \sim$ $1 / N$, significantly beyond the SQL. For instance, assuming an ensemble of $N$ spin-1 particles, according to the analysis above, the QCRB gives $\delta \kappa \geq \frac{1}{\sqrt{\nu}} \frac{1}{T N}$, and a proper initial probe state that saturates this bound is $\left(|j=1, m=1\rangle^{\otimes N}+|j=1, m=0\rangle^{\otimes N}\right) / \sqrt{2}$, which is nothing but the N-particle GHZ state, or the NOON state [34, 35], a cat state of spin-1 particles all in spin state $m=0$ or 1 .

The HL estimation of $\kappa$ in Eq. (12) with the NOON state discussed above also requires measurement of parity, i.e., $\hat{P}=(-1)^{a_{0}^{\dagger} a_{0}}$, where $a_{0}\left(a_{0}^{\dagger}\right)$ denotes the annihilation (creation) of a condensed particle in $m=0$ spin component. If a $\pi / 2$ rotation between $m=0$ and $m=1$ states is applied to all particles at once, we find $\langle\hat{P}\rangle_{\text {NOON }}=(-1)^{N / 2} \cos (N \kappa t)[36,37]$, whose unambiguous determination calls for single atom resolution in detecting $N$. In addition, as with the paired DFS entangled state of Eq. (7), NOON state with a meaningfully large $N$ remains to be generated. Were it to become available, such a Schrodinger cat state is known to be extremely fragile to the environment perturbations or imperfections of the control protocols $38-40]$.

Recent years have witnessed tremendous progresses in the generations of other forms of entangled ensembles with increasingly larger $N$ [30, 41, 42]. Compared to the state Eq. (7) suggested earlier, an analogous superposition based on the two particle DFS is

$$
\frac{1}{\sqrt{2}}\left(|m=1\rangle^{\otimes \frac{N}{2}}|m=-1\rangle^{\otimes \frac{N}{2}}+|m=0\rangle^{\otimes N}\right),
$$

which is also immune to stray magnetic fields. The above state reminds us of a superposition of two twin-Fock states Eq. (7), except for a subtle difference which might make it realizable in a spin-1 atomic condensate 30]. The two components of the superposition in Eq. (13), one twin-Fock state and one Fock state, can both be realized with quality, as the normal Fock state is simply the polarized state of all atoms in spin $(m=0)$ component.

Yet a more promising many particle entangled state for estimating $\kappa$ is the balanced atomic spin-1 Dicke state, 
which is readily available for experimental applications [14]. Spin squeezed [41, 43] will also work along the similar line of thoughts discussed below. Although spin- $1 / 2$ particles cannot be used because $\left(j_{z}^{(i)}\right)^{2}=1 / 4$ leading to a constant generator $\mathcal{H}=N / 4$, different $m$-states of higher spins (pseudo-spins) can be employed instead.

Dicke states are broadly defined as the eigenstates of collective spin or angular momentum $J^{2}\left(\vec{J}=\sum_{i} \vec{j}^{(i)}\right)$ and $J_{z}$. For a spin- 1 atomic condensate and assuming all spin components with the same spatial mode function, the balanced Dicke state with zero magnetization $\left(J_{z}=\right.$ 0 ) is described by the following wave function

$$
|D\rangle=\sum_{k=0}^{N / 2} 2^{(N-2 k) / 2} \sqrt{C_{N}^{k} C_{N-k}^{k} / C_{2 N}^{N}}|k, N-2 k, k\rangle,
$$

in the Fock state basis $|k, N-2 k, k\rangle$ of $k$ atoms each in $j_{z}^{(i)}=m= \pm 1$ and the other $N-2 k$ atoms in $j_{z}^{(i)}=m=0$ spin component [14]. In the notation of Eq. (7), the above basis state takes the form $|1,1\rangle^{\otimes k}|1,0\rangle^{\otimes(N-2 k)}|1,-1\rangle^{\otimes k}$ in terms of spin-1 eigenstate $|f=1, m\rangle . C_{N}^{k}$ denotes the combinatorial factor of choosing $k$ out of $N$. The balanced Dicke state possesses exceptional coherence [14]. Its basis states also forms a DFS, and is thus immune to linear Zeeman shifts from stray magnetic fields like states Eqs. (7) and (13).

The allowed precision limit for estimating $\kappa$ by the balanced Dicke state is shown in Fig. 1, where potential improvement over the SQL, or $-10 \log _{10}\left(N / \delta^{2} \kappa\right)$, is plotted in blue solid line. Although HL (red dashed line) is not saturated, its QFI scaling shown in Fig. 2 clearly supports an enhanced precision over the SQL, provided a suitable measurement scheme is found. Unfortunately, despite of our earnest effort, we have not been able to find an observable with its associate measurement protocol to saturate the QFI. If we follow the standard Ramsey interferometry and measure $J_{x}^{2}$ (or $J_{z}^{2}$ after a $\pi / 2$ rotation), we find a precision scaled as $\sim 1.48 / N^{0.46}$, which asymptotically approaches the SQL, but not beyond. Nevertheless, we consider this an encouraging result as single atom resolution in number counting is not required here. Furthermore, the structure of a DFS facilitates prolonged coherence time.

In more detail, the numerically computed QFI for estimating $\kappa$ by the balanced spin-1 Dicke state Eq. (14) is shown in Fig. 2] The fitted scaling exponent gives a $\gamma=1.94$ for $F_{Q} \propto N^{\gamma}$ at large $N$, which indicates that the ultimate precision for its estimation of $\kappa$ would asymptotically approach the HL.

Our proposal is equipped with at least two advantages: first it can be more readily applied to systems of large particle numbers; and second the suggested Dicke states are already available experimentally with more than 10000 atoms [14, 30]. According to results of Fig. 11. at $N=10^{4}$ atoms, the optimal improved measurement sensitivity for the LSV parameter $\kappa$ can be close to

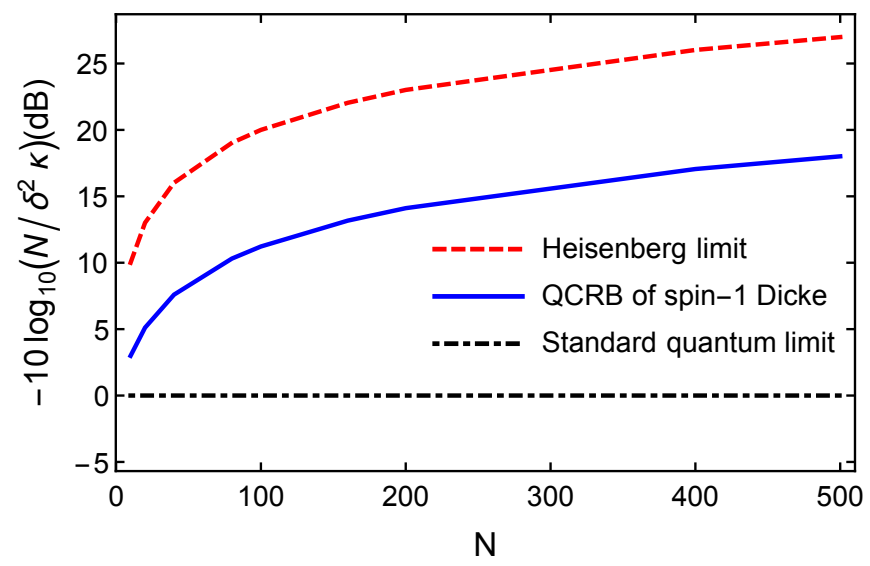

FIG. 1: The quantum Cramér-Rao bound (QCRB) for the balanced spin-1 Dicke state. The black dot-dashed line denotes SQL $(1 / \sqrt{N})$, while the red dashed line refers to the HL $(1 / N)$, saturable by NOON state with parity measurement. The blue solid line shows the QCRB of the balanced spin-1 Dicke state, which clearly implicates its ability for estimating $\kappa$ beyond the SQL.

2 (for SQL) to 4 (for HL) orders of magnitude smaller than experiments with two ions [10, 11]. The precision for the LSV parameter $C_{0}^{(2)}$ likewise can be improved potentially by the same orders of magnitude, according to the proportional relationship between the parameters $\kappa$ and $C_{0}^{(2)}$,

$$
\frac{\kappa}{2 \pi}=\frac{\left[\Delta E /\left(h C_{0}^{(2)}\right)\right]}{\Delta\left(j_{z}^{2}\right)} C_{0}^{(2)},
$$

which is obtained from Eq. (3) with $\Delta E\left(\Delta\left(j_{z}^{2}\right)\right)$ denoting the energy (angular momentum) deviation (fluctuation) for the experimentally selected states and $h$ is the Planck constant. For the balanced Dicke state we suggest with ${ }^{87} \mathrm{Rb}$ atoms, a rough estimate using the method of Ref. [11, 44] gives $\Delta E / h C_{0}^{(2)}=8.6 \times 10^{15} \mathrm{~Hz}$ and $\Delta\left(j_{z}^{2}\right)=1$ for the $m=1$ and $m=0$ states. Thus, assuming a year-long measurement with $10^{4}$ atoms, $\Delta \kappa \sim 10^{-9}$ can be expected in the SQL. This implies the parameter $C_{0}^{(2)}$ is bound at the level of $10^{-25}$, which is about two order of magnitude higher than the results reported previously [10, 11].

\section{CONCLUSION}

This paper discusses LSV effect for the directional dependent interaction (from the SME) of a single bound valence electron. A recent proposal for estimating its strength employing Ramsey interferometry is described, and possibilities of reaching enhanced estimation precision beyond the SQL in terms of entangled particles are studied. Two alternative but less complicated many particle entangled states are suggested, both capable of 


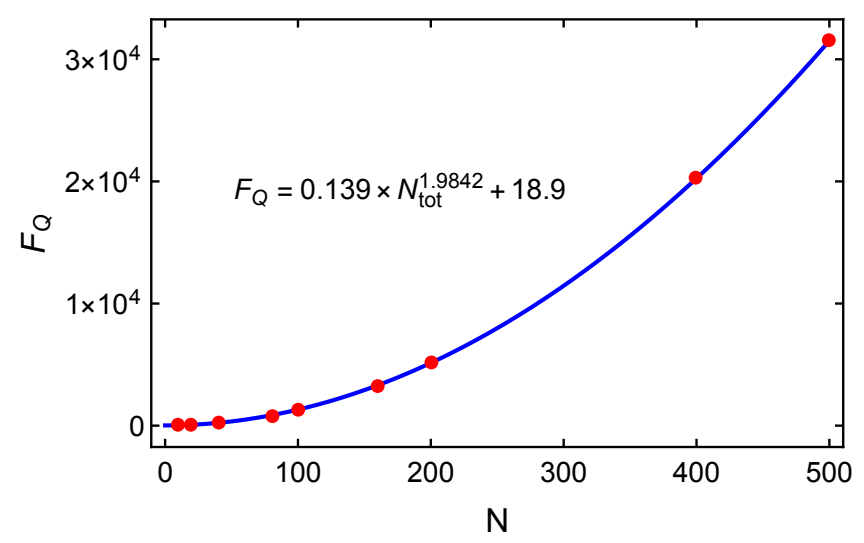

FIG. 2: A numerical fit to the QFI $\propto N^{\gamma}$ for the estimation of $\kappa$ by the balanced spin-1 Dicke state gives $\gamma \approx 1.98$, implicating that precision at the HL is possible.

reaching the precision of HL. Of particular relevance, we show that the balanced spin-1 Dicke state, which can be deterministically produced in spin-1 atomic BoseEinstein condensate of ${ }^{87} \mathrm{Rb}$ atoms [14], lives in a DFS, and can reach the precision of SQL by collective Ramsey interferometry without single atom counting resolution. Although its QFI implicates its ability for asymptotically reaching the HL precision, we have not been able to nail down an actual observable or measurement scheme for achieving this.

Finally, we note that there exist other LSV terms of the SME that reduce to interactions proportional to the z-component of Pauli operators [18], and thus are also testable using our proposed states. The details of how their associated energy level shifts arise may be different from our discussed Hamitonian (4), and deserve further investigations.

This work is supported by NSFC (No. 91636213 and No. 11654001). L.Y. also acknowledges the support of the National Key R\&D Program of China (Grant No. 2018YFA0306504) and the NSFC (Grant No. 11747605).
[1] D. Mattingly, Living Rev. Relativity 8, 5 (2005).

[2] S. Liberati, Class. Quantum Grav. 30, 133001 (2013).

[3] J. D. Tasson, Rep. Prog. Phys. 77, 062901 (2014).

[4] D. Colladay and V. A. Kostelecký, Phys. Rev. D 55, 6760 (1997).

[5] D. Colladay and V. A. Kostelecký, Phys. Rev. D 58, 116002 (1998).

[6] V. A. Kostelecký and N. Russell, Rev. Mod. Phys. 83, 11 (2011).

[7] S. Herrmann, A. Senger, K. Mohle, M. Nagel, E. V. Kovalchuk, and A. Peters, Phys. Rev. D 80, 105011 (2009).

[8] M. A. Hohensee, N. Leefer, D. Budker, C. Harabati, V. A. Dzuba, and V. V. Flambaum, Phys. Rev. Lett. 111, 050401 (2013).

[9] T. Pruttivarasin, M. Ramm, S. G. Porsev, et al., Nature 517, 592 (2015).

[10] V. A. Dzuba, V. V. Flambaum, M. S. Safronova, S. G. Porsev, T. Pruttivarasin, M. A. Hohensee, and H. Häffner, Nat. Phys. 12, 465 (2016).

[11] R. Shaniv, R. Ozeri, M. S. Safronova, S. G. Porsev, V. A. Dzuba, V. V. Flambaum, and H. Häffner, Phys. Rev. Lett. 120, 103202 (2018).

[12] C. W. Misner, K. S. Thorne, and J. A. Wheeler, Gravitation (Freeman, San Francisco, 1970).

[13] D. J. Wineland, J. J. Bollinger, W. M. Itano, F. L. Moore, D. J. Heinzen, Phys. Rev. A 46, R6797 (1992).

[14] Y. -Q Zou, L. -N Wu, Q. Liu, et al., Proceedings of the National Academy of Sciences 115, 6381 (2018).

[15] H. Muller, S. Herrmann, A. Saenz, A. Peters, and C. Lammerzahl, Phys. Rev. D 70, 076004 (2004).

[16] V. A. Kostelecký and A. J. Vargas, Phys. Rev. D 92, 056002 (2015).

[17] R. Bluhm, V. A. Kostelecký, and N. Russell, Phys. Rev. Lett. 82, 2254 (1999).

[18] V. A. Kostelecký and C. D. Lane, Phys. Rev. D 60, 116010 (1999).

[19] V. A. Kostelecký and C. D. Lane, J. Math. Phys. (N.Y.) 40, 6245 (1999).
[20] V. A. Kostelecký and J. D. Tasson, Phys. Rev. D 83, 016013 (2011).

[21] D. A. Lidar and T. A. Brun, Quantum Error Correction, (Cambridge University Press, Cambridge, England, 2013).

[22] N. Ramsey, Molecular beams, (Oxford University Press, Oxford, UK, 1985).

[23] B. Yurke, S. L. McCall, and J. R. Klauder, Phys. Rev. A 33, 4033 (1986).

[24] M. Kitagawa and M. Ueda, Phys. Rev. A 47, 5138 (1993).

[25] R. G. Unanyan and M. Fleischhauer, Phys. Rev. Lett. 90, 133601 (2003).

[26] S. Choi and N. P. Bigelow, Phys. Rev. A 72, 033612 (2005).

[27] S. Choi and B. Sundaram, Phys. Rev. A 77, 053613 (2008).

[28] C. W. Helstrom, Quantum detection amd estimation theory, (Academic Press, New York, 1976).

[29] M. G. Paris, International Journal of Quantum Information 7 (supp01), 125 (2009).

[30] X. Y. Luo, Y. Q. Zou, L. N. Wu, et al., Science 355, 620 (2017).

[31] S. L. Braunstein and C. M. Caves, Phys. Rev. Lett. 72, 3439 (1994).

[32] S. L. Braunstein, C. M. Caves, and G. J. Milburn, Ann. Phys. 247, 135 (1996).

[33] S. Boixo, A. Datta, S. T. Flammia, A. Shaji, E. Bagan, and C. M. Caves, Phys. Rev. A 77, 012317 (2008).

[34] A. N. Boto, P. Kok, D. S. Abrams, S. L. Braunstein, C. P. Williams, and J. P. Dowling, Phys. Rev. Lett 85, 2733 (2000).

[35] H. Lee, P. Kok, and J. P. Dowling, Journal of Modern Optics 49, 2325 (2002).

[36] T. Kim, O. Pfister, M.J. Holland, J. Noh, and J.L. Hall, Phys. Rev. A 57, 4004 (1998).

[37] C. C. Gerry and J. Mimih, Contemporary Physics 51, 497 (2010).

[38] S. F. Huelga, C. Macchiavello, T. Pellizzari, and A. K. 
Ekert, M. B. Plenio, and J. I. Cirac, Phys. Rev. Lett 79, 3865 (1997).

[39] B. M. Escher, R. L. de Matos Filho, and L. Davidovich, Nat. Phys. 7, 406 (2011).

[40] U. Dorner, R. Demkowicz-Dobrzanski, B. J. Smith, J. S. Lundeen, W. Wasilewski, K. Banaszek, and I. A. Walmsley, Phys. Rev. Lett 102, 040403 (2009).

[41] O. Hosten, N. J. Engelsen, R. Krishnakumar, et al., Na- ture 529, 505 (2016).

[42] J. G. Bohnet, B. C. Sawyer, J. W. Britton, et al., Science 352, 1297 (2016).

[43] K. C. Cox, G. P. Greve, J. M. Weiner, and J. K. Thompson, Phys. Rev. Lett 116, 093602 (2016).

[44] V. V. Flambaum, Phys. Rev. Lett. 117, 072501 (2016). 